\title{
A practical solution to a newspaper distribution problem
}

\author{
R.J. Mantel and M. Fontein \\ University of Twente, Department of Mechanical Engtneering, Production and Operations Management Group, \\ P.O. Bo. 217, 7500 AE Enschede. The Netherlands
}

\begin{abstract}
In this paper the problem of distributing newspapers is treated. After a general introduction on this topic, a mathematical model for a hierarchical distribution system is given explicitly and a heuristıc consistıng of several solution techniques is described. Furthermore. some results of the application of this heuristic in a study performed for a Dutch regional newspaper are presented and discussed There is also an indication as to how the approach could be improved
\end{abstract}

\section{Introduction}

Nowadays efficient distribution is seen as an important critical success factor by many newspaper companies. On the one hand, management wishes the papers to be printed as late as possible in order to include the most recent news, while on the other hand, the reader likes to receive his newspaper as early as possible. A good distribution system may help to solve this problem of apparently conflicting interests in an acceptable way. Here, indeed, time rather than costs, is the critical factor. The efficiency of the distribution system may strongly affect the competitiveness of a newspaper.

This contribution deals with a study performed for a Dutch regional newspaper with 140000 papers printed per day, a number which is growing rapidly. It appears 6 days a week and there are eight different editions, each containing news for a particular region. The total distribution area is relatively large with many subareas in which the population density is very low.

Correspondence to: R.J. Mantel and M. Fontein. University of Twente, Department of Mechanical Engineering, Production and Operations Management Group, P.O. Box 217, 7500 AE Enschede, The Netherlands
The reason management wishes to perform the study reported here is the fact that suboptimal expansion decisions in the past have led to an inefficient distribution system. There is a great need for a decision support tool to evaluate and improve the distribution system. What--if questions to be investigated by such a tool may include:

- Is the truck fleet still adequate?

- What are the consequences of different strategies, e.g. more distribution by company-owned trucks in order to be less dependent on hired transport facilities?

- What is the relation between distribution time and costs? Is it possible to reduce both at the same time?

The tool must be flexible enough because, e.g., edition's region boundaries may change. The outline of the remainder of this paper is as follows. In Section 2 the newspaper distribution process is described, for which subsequently in Section 3 a mathematical model is formulated. Section 4 deals with solution techniques and in Section 5 results for a part of the physical distribution network are presented. Finally, some concluding remarks are given in Section 6.

The distribution system consists of several hierarchical levels. This implies a kind of 
problem, mostly referred to as "hub and spoke" or location-routing problems, on which little literature is available. A related paper on this subject was produced by Jacobsen and Madsen [1] and a paper by Laporte et al. [2] should also be mentioned. Graph theory and vehicle routing is extensively treated in Refs. [3-5].

\section{The distribution process}

The different editions are printed one after another and consequently they are distributed separately; in most cases more than one edition at the same time. Obviously, the printing sequence determines the distribution sequence. As soon as an edition is printed the shipping department counts the papers needed for the various delivery nodes and packs them in foil. Next, the papers are put into a truck in the correct delivery order, after which they are ready for distribution.
The complete distribution system for each edition's region consists of three hierarchical levels, as illustrated in Fig. 1.

Level 1: First, large company-owned trucks transport the papers from the central shipping node to transfer nodes. This transport is carried out each day by one or two trucks in parallel according to fixed delivery schemes. Only on Saturdays the are schemes different, since on these days the number of pages in a paper is considerably larger causing much higher transportation weights and volumes.

Level 2: From the transfer nodes the papers are further distributed to the delivery nodes by hired drivers with private passenger cars. Hence, on this level we may also speak of parallel distribution. The routes travelled by the passenger cars will be referred to as "subroutes".

Level 3: In the delivery nodes newsboys pick up the papers to carry out the final stage of the distribution process, i.e. transport to the subscribers.

It should be noted that the real distribution system is even more complex than outlined

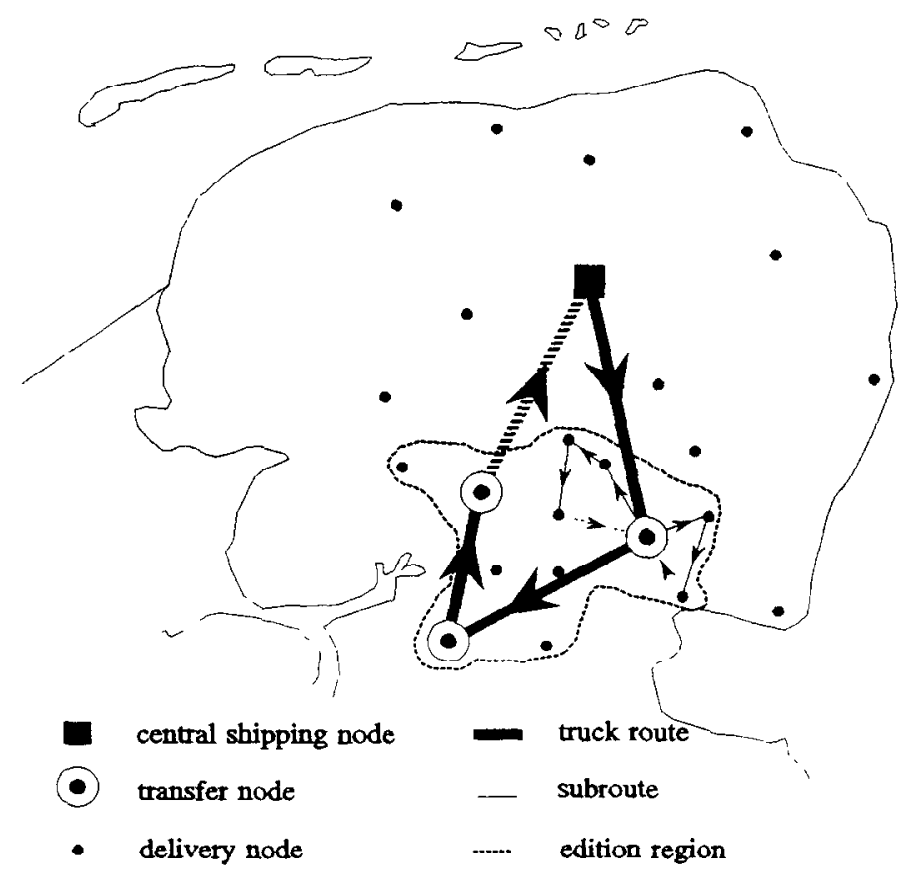

Fig. 1. The hierarchical distribution system. Note: the dashed link in a route indicates empty travel. 
above because, e.g., kiosks and petrol stations also have to be supplied.

\section{Model description}

The distribution process described in the preceding section is translated into a model in which level 3 is not taken into account, because this level

- concerns a rather short process and so there are not many opportunities to save time there,

- involves relatively low costs and

- is hard to control. Here, - in contrast to level $1,-$ it is difficult to require fixed schedules. Owing to its nature the distribution system is represented as a network model. The variables in the model are:

- The number of transfer nodes Increasing this number may lead to

- longer total travelling time for trucks,

- shorter total travelling time for passenger cars and

- smaller amounts of papers to be transshipped in each transfer node.

- the locations of transfer nodes

These locations also determine the ratio between total truck travelling time and total travelling time of passenger cars. A satisfactory balance between both times should be established.

- the number and routing of trucks

- the number and routing of passenger cars.

Fixed input data are:

- mean travelling speeds on different roads, determined by speed limits,

- the road network and the distances between adjacent nodes from which, together with the speed data, the travelling times between these nodes can be calculated,

- times to load and unload and

- the capacities of trucks and passenger cars. These capacities determine to a great extent the number of routes to be travelled and so the number of vehicles needed. The truck capacities can only be changed in the long term. The capacities of the passenger cars are fixed because, due to legal rules, the loading of passenger cars is limited.

The objective is to minimize total travclling time because, as stated in the introduction, time is the most important factor. Moreover, total travelling time reflects to a great extent the direct costs, as expenses for petrol and allowances for the hired drivers. Hence, the strongly interdependent total travelling times of trucks and passenger cars are minimized. This is done subject to capacity constraints for trucks and passenger cars and constraints on the total time available for distribution, i.e. the time between the moment the paper is ready for distribution and the latest moment the paper has to be delivered to a subscriber. As mentioned before, the printing sequence of subsequent editions determines when to distribute which edition. Therefore, the time available for distribution is different for each edition's region. As level 3 is not modelled, the distribution time needed for this level must be taken into account to obtain the net distribution time available for levels 1 and 2 .

Now the problem may be mathematically formulated. First it follows a description of the variables used.

$i, j, q$ are delivery nodes

$i=0 \quad$ is the central shipping node

$l \quad$ a truck

$s \quad$ a passenger car

$Q \quad$ demand for papers in node $i$

$N \quad$ set of all delivery nodes

$S \quad$ set of transfer nodes, i.e. a subset of $N$

$S^{\mathrm{c}} \quad=S \cup\{i=0\}$

$|X| \quad$ cardinality of set $X$

$m_{1} \quad$ number of trucks

$m_{2} \quad$ number of passenger cars

$t_{i j} \quad$ travelling time from $i$ to $j$

$X_{i j l}=1$ if arc $(i, j)$ is travelled by truck $l$ and equal to 0 otherwise

$Z_{l j s} \quad$ idem for passenger car $s$

a maximum total distribution time

$C_{1} \quad$ capacity of a truck

$C_{2} \quad$ capacity of a passenger car

$R_{i} \quad$ amount of papers delivered in transfer node $i$ for distribution along all subroutes starting in $i$

$R_{i s} \quad$ part of $R_{\imath}$ carried by $s$ 
$H_{s}$ amount of papers carried by $s$

$\tau_{\imath} \quad$ time to travel by a truck from the central shipping node to node $i$

$f$ time to load each paper (is determined from the time to load a bundle of papers)

$e \quad$ time to unload each paper (is determined from the time to unload a bundle of papers)

$M \quad$ a very large number

$Y_{i} \quad$ a variable assigned to node $i$

The distribution problem for each edition's region may be described by the mixed integer problem given below:

$P^{* *}=\min _{m_{1}, m_{2}} P^{*}$,

which implies that for all possible combinations of $m_{1}$ and $m_{2}$ the optimal set of transfer nodes $(S)$ must be determined:

$P^{*}\left(m_{1}, m_{2}\right)=\min _{\varsigma \in N} P\left(m_{1}, m_{2}, S\right)$.

where

$$
\begin{aligned}
P\left(m_{1}, m_{2}, S\right)= & P\left(m_{1}, m_{2}, S^{0}\right) \\
= & \min _{X_{1, l} . Z_{i, j}} \sum_{t \in S^{0}} \sum_{j \in S^{0}} \sum_{i=1}^{m_{1}} X_{i j l} t_{\imath \jmath} \\
& +\sum_{i \in N} \sum_{j \in N} \sum_{s=1}^{m_{2}} Z_{i j s} t_{i j}
\end{aligned}
$$

subject to

$$
\begin{aligned}
& \sum_{i \in S^{0}} \sum_{l=1}^{m_{1}} X_{i j l}=1, \quad j \in S, \\
& \sum_{j \in S^{0}} \sum_{l=1}^{m_{1}} X_{i j l}=1, \quad j \in S, \\
& \sum_{i \in S^{0}} X_{i q l}-\sum_{j \in S^{\circ}} X_{q j l}=0, \quad \forall q, 1, \\
& \sum_{l \in S}\left(Q_{l}+R_{l}\right) \sum_{j \in S^{0}} X_{l j l} \leqslant C_{1}, \quad \forall l,
\end{aligned}
$$

$$
\begin{aligned}
\tau_{j} \geqslant & \tau_{i}+\sum_{l=1}^{m_{1}} X_{l j l}\left[t_{i j}+\left(Q_{l}+R_{l}\right) e\right] \\
& -M\left(1-\sum_{l=1}^{m_{1}} X_{\imath \jmath l}\right), \quad i \in S^{0}, \quad j \in S,
\end{aligned}
$$

$$
\tau_{0}=0
$$

$$
\begin{aligned}
\sum_{i \in S} \sum_{J \in N-S}\left[Z_{i j s} \tau_{\mathrm{t}}+R_{i s}(f+e)\right] & \\
+\sum_{i \in N} \sum_{j \in N-S} Z_{i j s} t_{\imath \jmath} & \leqslant a, \\
s & =1, \ldots, m_{2},
\end{aligned}
$$

$\sum_{\imath \in S} X_{t 0 l}=1, \quad l=1, \ldots, m_{1}$,

$\sum_{j \in S} X_{0 \jmath l}=1, \quad l=1, \ldots, m_{1}$,

$$
\begin{gathered}
Y_{i}-Y_{j}+|S| \sum_{l=1}^{m_{1}} X_{i j l} \leqslant|S|-1 \\
i \neq j, \quad i \in S^{0}, \quad j \in S^{0}
\end{gathered}
$$

$\sum_{\imath \in N} \sum_{s=1}^{m_{2}} Z_{1, j s}=1, \quad j \in N-S$,

$$
\sum_{j \in N} \sum_{s=1}^{m_{2}^{2}} Z_{i j s}=1, \quad i \in N-S,
$$

$$
\sum_{i \in N} Z_{\imath q s}-\sum_{j \in N} Z_{q \jmath s}=0
$$

$$
\begin{gathered}
q \in N-S, \quad s=1, \ldots, m_{2}, \\
H_{s}=\sum_{i \in N-S} Q_{i} \sum_{j \in N} Z_{i j s} \leqslant C_{2}, \\
s=1, \ldots, m_{2}, \\
R_{i s}=\sum_{i \in N-s} Z_{i j s} H_{s} \quad i \in S, \quad s=1, \ldots, m_{2},
\end{gathered}
$$

$$
R_{\imath}=\sum_{s=1}^{m_{2}} R_{i s .} \quad i \in S
$$


$\sum_{i \in S} \sum_{j \in N-s} Z_{i j s}=1, \quad s=1, \ldots, m_{2}$

$\sum_{j \in S} \sum_{\imath \in N-s} Z_{i j s}=1, \quad s=1, \ldots, m_{2}$

$Y_{i}-Y_{j}+|N| \sum_{s=1}^{m_{2}} Z_{i j s} \leqslant|N|-1$,

$i \neq j, i \in N-S, j \in N-S$,

$X_{\imath \jmath l}=0,1, \quad \forall i, j, l$,

$Z_{i j s}=0,1, \quad \forall i, j, s$,

$y_{i}$ arbitrary, $\forall i$.

Constraints (4) and (5) ensure that each transfer node is visited exactly once by a truck, while (14) and (15) ensure that each of the other delivery nodes is visited exactly once by a passenger car. Constraint (6) implies that a truck which enters a transfer node also leaves that node, while (16) is a similar constraint for passenger cars and the other delivery nodes. The capacity limits for trucks and passenger cars are reflected in (7) and (17), respectively.

Inequality (10) (together with (8) and (9)) constitutes the time constraint. Equations (11) and (12) ensure that all trucks leave and enter the central shipping node only once, while (20) and (21) ensure that all passenger cars leave and enter a transfer node only once. Constraint (13) is a subtour elimination condition by Miller et al. [6] for the transfer nodes and (22) is the same condition for the other delivery nodes. Finally, constraints (18) and (19) are needed to determine transfer amounts of papers needed in other constraints; in (18) the amount of papers transferred via node $i$ is computed for each passenger car separately and next in (19) these amounts are summed over all passenger cars in order to obtain the total amount of papers transferred via node $i$. Due to (18) the problem is non-linear.
It is possible to include the time to transship the papers in the transfer nodes in the objective function. This is done here, the results of which are reported in Section 5. The transfer time varies with the size of $S$; if there are no transfer nodes and if all delivery nodes are transfer nodes then this time is zero. Somewhere between these two extremes the total transfer time reaches its maximum value.

The formulation above clearly demonstrates that the problem is far too complex to compute the exact optimal solution. It contains several interdependent problems such as:

- What are the best locations of the transfer nodes?

- What is the optimal order of visiting these transfer nodes in a truck route? In this respect it should be noted that, since the maximum total distribution time is equal for all delivery nodes, the radius of action of passenger cars from the first transfer nodes in a truck route is larger (i.e. the distribution time remaining for subroutes is longer) than from the latter ones. Related to this:

- Which delivery nodes are assigned to (supplied by) which transfer nodes? A larger number of nodes can be supplied from the first transfer nodes in a truck route than from the latter ones.

To illustrate this, the effect of the time constraints is shown in Fig. 2. The radius of action from transfer nodes near the central shipping node is larger than for transfer nodes further away. Furthermore, in this figure the effect of the passenger car capacity constraints - leading to an equal action radius for each transfer node is indicated, which partly extinguishes the effect of the time constraints.

For the first transfer nodes in a truck route, the capacity constraint is the limiting factor while the time constraint is not limiting, whereas for the latter transfer nodes in such a route the opposite holds.

It may be stated that the complexity of the problem is caused by the hierarchical structure of the system and the strong interactions between the levels of this structure. Therefore, to simplify the problem, constraint (10) is 


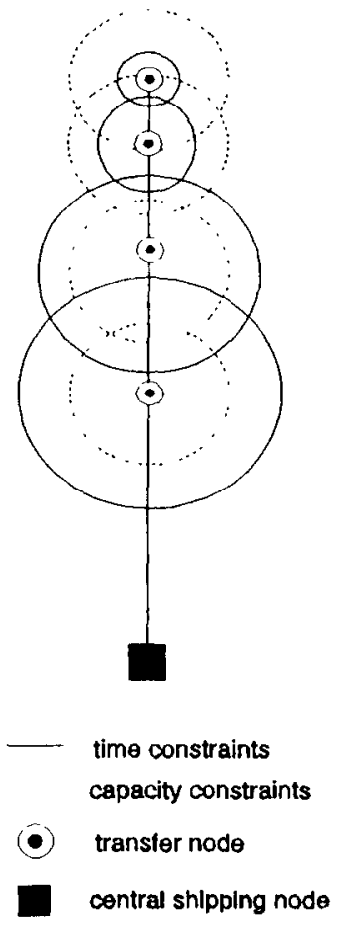

Fig. 2. Action radius due to time and capacity constraints for subroutes from transfer modes in a truck route.

replaced by the following two constraints:

$\sum_{\imath \in S^{\circ}} \sum_{j \in S} X_{i j l}\left[t_{i j}+\left(Q_{\imath}+R_{\imath}\right) e\right] \leqslant a_{1}$

$$
\begin{gathered}
l=1, \ldots, m_{1} \\
\sum_{i \in S} \sum_{j \in N-S} R_{\imath s}(f+e)+\sum_{i \in N} \sum_{j \in N-S} Z_{i j s} t_{t j} \leqslant a_{2} \\
s=1, \ldots, m_{2}
\end{gathered}
$$

where, in addition to the symbols given before,

$a_{1}$ is the maximum duration of a truck route and $a_{2}$ the maximum duration of a subroute $\left(a_{2}=a-a_{1}\right)$.

So the problem is split into two independent parts. In this formulation the maximum duration of a subroute is assumed to be equal for all subroutes and, consequently, the number of delivery nodes supplied by a transfer node will be more or less the same for all transfer nodes.

Further, the capacities of the trucks appear to be such that all papers for one edition's region fit into one truck and so the truck capacity constraints are ignored. The heuristic, described in the next section, is based on the latter formulation.

\section{Solution techniques}

The heuristic described is a combination of several mostly well-known methods. These standard methods are described in the appendix. As usual, for this kind of problems, the input available consists of a set of basic data, among which the travelling times between all pairs of adjacent nodes. From this, the complete travelling time matrix, containing the travelling times between all pairs of nodes in an edition's region, is easily constructed by using the algorithm of Floyd [7].

Next, in each edition's region the best locations for $k$ transfer nodes, with $k=1, \ldots, n$ (where $n$ is the total number of delivery nodes in the region, i.e. $|N|$ ), and the allocation of delivery nodes to these transfer nodes are determined. This is done by an adapted heuristic for location-allocation problems in networks [8]. The adaptation implies that instead of visiting all delivery nodes directly from the transfer node, tours are now constructed along these nodes by a vehicle routing heuristic (the well-known saving technique developed by Clarke and Wright [9]). Also the truck routes from the central shipping node along the $k$ transfer nodes are determined by means of the Clarke and Wright heuristic. The complete procedure for one edition's region, which resembles the ALA-SAV method in [1] (because it also applies a location-allocation and a saving technique), is as follows:

For $k=1, \ldots, n$ the following steps are performed:

(1) Select $k$ arbitrary transfer nodes from the complete set of $n$ delivery nodes. 
(2) Allocate each delivery node to its nearest transfer node. In this way the $n$ nodes are subdivided into $k$ subsets of nodes.

(3) Determine for each subset, created in step 2 , the best location for the transfer node among all nodes in the subset. Hence, the location of the transfer node may change within the subset. This is done by determining, successively for each node in the subset, the subroutes along all other nodes in the subset by means of the Clarke and Wright heuristic, taking both time and capacity constraints into account. The node which involves the shortest total travelling time of passenger cars for the subset is selected as the new transfer node. If the locations of all $k$ transfer nodes are unchanged compared to the previous $k$ locations, then the investigations for $k$ transfer nodes are finished, unless step 4 has not been carried out before for $k$ transfer nodes. In the latter case only step 4 must be carried out, after which the investigations for $k$ transfer nodes are finished.

If the locations did change then step 4 must be carried out and next return to step 2.

(4) Determine the most efficient routing of the trucks along all transfer nodes found in step 3 , again by using the Clarke and Wright heuristic and now taking the time constraint for truck routes into account. Add the total truck travelling time to the sum of travelling times on the subroutes (Note: a subroute starts from a transfer node as soon as a truck has visited this transfer node) as found in step 3. If the result is better than for any situation with $k$ transfer nodes investigated so far then all information concerning the routing scheme (locations of transfer nodes and the routing of trucks and passenger cars) is saved as the best solution for $k$ transfer nodes found so far.

\section{Results}

The heuristic described in the preceding section results, for $k=1, \ldots, n$, in the best found transfer node locations and routing schemes for trucks and passenger cars. For a given edition's region (with 60 delivery nodes, $a_{1}$ and $a_{2}$ set to 4 and $2 \mathrm{~h}$ respectively, a passenger car capacity of 1100 papers) the results are presented in Fig. 3, in which several functions are shown:

- total truck travelling time,

- total travelling time on subroutes,

- sum of both times,

- longest total distribution time faced by a subscriber.

The straight vertical dashed lines in the figure indicate where, due to the time constraint, an additional truck is needed. From the figure it may be concluded that for the simplified problem total travelling time is rather insensitive
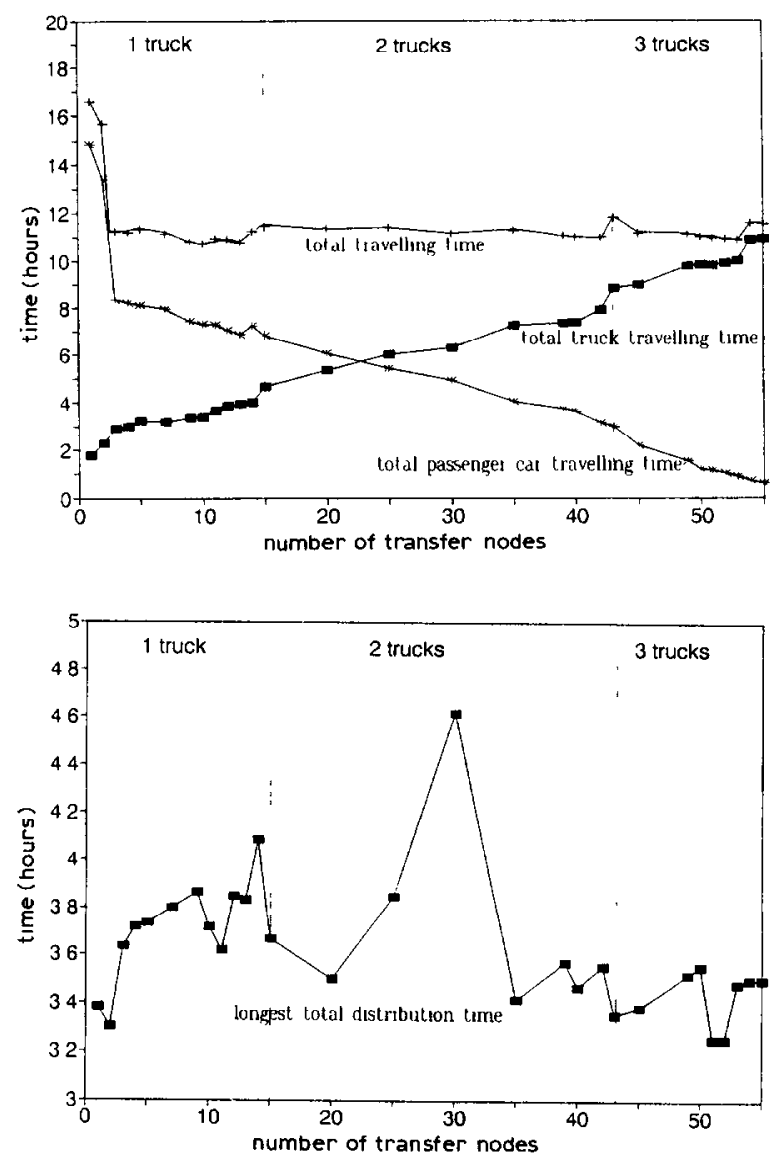

Fig. 3. Total travelling times and longest total distribution time as functions of the number of transfer nodes in an edition's region. 
to the number of transfer nodes; for a large range the total travelling time is not much higher than the minimal value, which amounts to $10 \mathrm{~h}$ and $42 \mathrm{~min}$ and occurs for 10 transfer nodes. The effect of the total transfer time, as stated in Section 3, is possibly one of the causes for this flat function. It is hard to say if the simplification of the original problem by splitting it into two independent subproblems by constraints (26) and (27) is also a cause. Both for a low number of transfer nodes total travelling time increases due to (27) and for a high number of transfer nodes this time increases due to (26) when compared to these situations without these constraints.

Owing to the flat function, secondary criteria as such the number of trucks used or a maximum fraction of passenger car travelling time, rather than the total travelling time, should be used. The peak in the curve of the longest total distribution time can be explained as follows. If there are no transfer nodes it means that no trucks are used and all transportation is carried out by passenger cars. Hence, in this case one may speak of a onelevel distribution problem and, consequently, only the time constraint for passenger cars (27) needs to be considered (so constraint (26) may be ignored); therefore, the maximum total distribution time is only $a_{2}$. If the number of transfer nodes is equal to the total number of delivery nodes in the region then the opposite situation occurs, i.e. only transportation by trucks, with a maximum total distribution time of $a_{1}$. Only if the number of transfer nodes is approximately equal to half the number of delivery nodes then one may speak of a two-level distribution problem with a maximum total distribution time of $a_{1}+a_{2}$ and, consequently, there the graph shows a peak.

\section{Concluding remarks}

The newspaper distribution problem is mathematically formulated, among other things, in order to know all the aspects of the problem. Next, in order to solve it, the mathematical model was simplified by an approx- imation with respect to the time constraint. This simplified problem was then solved heuristically. Therefore, the study reported in this paper should be considered as a first attempt to solve the original problem. Especially, the strong hierarchical interdependence between truck routes and subroutes should be handled in a correct way; as already stated, the maximum duration $\left(a_{2}\right)$ of a subroute from a transfer node shoud not be constant but a variable that increases if the position of the node in the truck route is nearer to the central shipping node. Probably, the mathematical formulation presented in this paper can lead to an improved heuristic and to a method for setting lower bounds for the objective function. Nevertheless, the approach presented can already be used as a decision support tool for the planners; it may be used as a basis for a structured solution to the newspaper distribution problem.

Other topics for further research include

- the interaction between the different editions, i.e. optimization of the printing sequence of the editions, and

- the improvement of the routing schemes by applying more advanced vehicle routing techniques.

\section{Appendix: Short description of the standard methods applied}

Floyd's method

Given a network and the distances between all adjacent nodes in this network, Floyd's (exact) method determines the distances between all pairs of nodes by applying the following recursive expression:

$$
d_{i j}^{m}=\min _{k}\left(d_{i j}^{m-1}, d_{i k}^{m-1}+d_{k j}^{m-1}\right)
$$

for all $i, j=1, \ldots, n$ and $k=1, \ldots, n$, and where $d_{i j}^{0}$ is equal to the distance between $i$ and $j$ if there is an arc between these nodes and set to infinite otherwise.

As soon as the values of all variables in a certain iteration are the same as those in the previous iteration then the algorithm stops. 


\section{Location-allocation problem in a network}

Given a network in which the nodes represent customers and given the supply frequencies of these customers, the problem is to locate a number $(n)$ of distribution centers, from which customers are supplied directly, in $n$ of the nodes and to allocate each customer to one of these distribution centers such that the total distribution time is minimized.

A heuristic to solve this problem is as follows. Select $n$ arbitrary nodes as distribution centers and allocate each customer to its nearest distribution center, which results in $n$ clusters of nodes. Next determine, by full enumeration, for each cluster the optimal location of the distribution center within the cluster. If the locations of all centers remain unchanged then the procedure may be ended, else allocate each customer again to its nearest distribution center and repeat the procedure.

As this method is a heuristical approach, in most cases the result will not be optimal. Therefore, it is recommended to execute the heuristic several times with different initial locations of the distribution centers.

\section{The Clarke and Wright savings method}

The method of Clarke and Wright is a simple heuristic to solve a vehicle routing problem with one distribution center and a number of customers, whose demands are given. Also the travelling times between the nodes must be known. Several constraints such as capacity and time limits can easily be handled. The heuristic is as follows. First, com- pute for each pair of customers the travelling time saved by visiting both customers in a subtour from and to the distribution center instead of visiting both customers separately. Then all pairs must be placed in a decreasing saving order, in which the pairs of customers are then linked together into subtours, if the constraints allow so.

\section{References}

[1] Jacobsen, S.K. and Madsen. O.B.G., 1980. A comparative study of heuristics for a two-level routinglocation problem. Eur. J. Oper. Res., 5: 378-387.

[2] Laporte. G., Nobert, Y. and Taillefer, S., 1988. Solving a family of multi-depot vehicle routing and location-routing problems. Transp. Sc1., 22: 161-172.

[3] Christofides, N., Graph Theory: An Algorithmic Approach. Academic Press, London.

[4] Lawler, E.L., Lenstra, J.K., Rinnooy Kan, A.H.G. and Shmoys, D.B., The Traveling Salesman Problem. Wiley, Chichester.

[5] Papadrimitriou. C.H. and Steiglitz, K., Combınatorial Optimization: Algorithms and Complexity. Prentice-Hall, Englewood Cliffs, NJ.

[6] Miller, C., Tucker, A.W. and Zemlin, R.A., 1960. Integer programming formulation of the travelling salesman problem. J. ACM, 7: 326-329.

[7] Floyd, R.W., 1967. Algorithm 97, shortest path. Commun. ACM, 5.

[8] Francis, R.L. and White, J.A., Facility Layout and Location. Prentice-Hall, Englewood Cliffs, NJ.

[9] Clarke, G. and Wright, J.W., 1964. Scheduling of vehicles from a central depot to a number of delivery points. Oper. Res., 12: $568-581$.

[10] Kusiak, A., 1983. Scheduling of vehicles: Algoruthms. Proc. 5th Int. Conf. on Automation in Warehousing, Atlanta. USA. 